\title{
A linear-elasticity solver for higher-order space-time mesh deformation
}

\author{
Laslo T. Diosady*and Scott M. Murman ${ }^{\dagger}$ \\ NASA Ames Research Center, Moffett Field, CA, USA
}

\begin{abstract}
A linear-elasticity approach is presented for the generation of meshes appropriate for a higher-order space-time discontinuous finite-element method. The equations of linearelasticity are discretized using a higher-order, spatially-continuous, finite-element method. Given an initial finite-element mesh, and a specified boundary displacement, we solve for the mesh displacements to obtain a higher-order curvilinear mesh. Alternatively, for moving-domain problems we use the linear-elasticity approach to solve for a temporally discontinuous mesh velocity on each time-slab and recover a continuous mesh deformation by integrating the velocity. The applicability of this methodology is presented for several benchmark test cases.
\end{abstract}

\section{Introduction}

In our recent work we have been developing higher-order space-time finite-element methods for turbulent compressible flows. ${ }^{1-5}$ Higher-order methods can provide greater efficiency for simulations requiring high spatial and temporal resolution than traditional second-order computational fluid dynamics (CFD) methods. We have shown the advantage of using higher-order methods for compressible turbulent flows ${ }^{1,2,4}$ and our desire is to take advantage of higher-order methods for the simulation of coupled fluid/structures interaction problems. ${ }^{6}$

In a space-time formulation the entire four-dimensional (three-spatial + time) space-time domain is discretized using finite-elements with polynomials in both space and time. ${ }^{7-13}$ For fixed-domain simulations, the spatial mesh is simply extruded in the temporal direction to form the space-time mesh. However, a space-time finite-element formulation can be naturally extended for moving-domain problems by deforming the spatial boundaries of the space-time mesh as a function of time. By treating the spatial and temporal discretization in a unified manner, the resulting discretization guarantees the satisfaction of the geometric conservation law (provided sufficient integration is used). ${ }^{14}$ This has made the space-time formulations a natural choice for moving domain and FSI simulations. ${ }^{7,8,12,13}$

A necessary component for moving-body simulations is the generation of the space-time finite-element mesh. Oftentimes the space-time mesh may correspond to multiple "slabs", based on either a fixed spatial topology $8,11,12$ or varying spatial topologies connected through an unstructured space-time triangulation. ${ }^{13}$ Alternatively, a fully-unstructured space-time meshing approach may be used. ${ }^{15}$ In this work we initially consider a slab-based approach where a fixed spatial mesh topology is assumed and the space-time mesh is formed by extruding this mesh in the temporal direction while modifying the location of mesh nodes in order to conform to a given boundary displacement. In particular we use a linear-elastic analogy to move the mesh nodes which has previously been used for low-order space-time finite-element simulations. ${ }^{12,16}$ While there are a variety of different approaches which may be used for mesh deformation, we choose the elasticity approach as it fits naturally into our existing finite-element framework. In this work we extend the elasticity approach to the generation of higher-order space-time meshes.

The use of higher-order methods necessitates using a higher-order representation of the curved boundaries. ${ }^{17,18}$ For fixed-domain simulations, this implies that the boundary elements are curved to match the true geometry. The elastic analogy has also been widely used for the curving of meshes for steady-state

*Science and Technology Corp, laslo.diosady@nasa.gov

†Scott.M.Murman@nasa.gov 
or fixed-grid simulations. ${ }^{19-22}$ Previously, a non-linear elasticity approach was used for obtaining mesh displacements for a higher-order discontinuous-Galerkin arbitrary Lagrangian-Eulerian (ALE) method. ${ }^{22}$ In this approach the elasticity solver was employed to solve for the mesh displacements at each stage of an implicit Runge-Kutta scheme, while the mesh velocities were computed from the displacements in a manner consistent with the temporal discretization. In this work we employ a similar approach, however we solve directly for the 4-dimensional velocity field for each time slab and compute the displacement as the temporal integral of this field. In the case of a fixed temporal order and an appropriate quadrature rule there is an equivalence between this method and the implicit RK approach previously employed. However, the current approach allows for the additional flexibility of allowing different temporal orders to be used in different elements. Additionally, this approach was chosen since it fits well into our existing space-time framework allowing the same solver to be used for solving unsteady structural mechanics problems.

The remainder of this paper is as follows. In Section II we describe in detail the discretization of the linear-elastic problem and briefly discuss the solution strategy. In Section III we present numerical results using this methodology. In Section IV we present initial coupling of the mesh deformation with our existing fluid solver. Finally we conclude with a summary and discuss future outlook in Section V.

\section{Discretization}

We solve the equations of linear elasticity to obtain the volume displacement of the fluid mesh given the prescribed motion of the surface (or part of the surface) of the fluid mesh. The equations of linear elasticity are:

$$
\mathbf{v}_{, t}-\sigma_{i j, j}=0
$$

where $\mathbf{v}=\boldsymbol{u}_{, t}$ is the velocity field, while $\boldsymbol{u}$ is the displacement field. Here $\sigma_{i j}$ is the Cauchy stress tensor given by:

$$
\sigma_{i j}=2 \mu \epsilon_{i j}+\lambda \epsilon_{k k}
$$

where $\mu=\frac{E}{2(1+\nu)}$ and $\lambda=\mu \frac{2 \nu}{1-2 \nu}$ are the Lamé constants given as a function of the Young's Modulus, $E$, and Poisson ratio, $\nu$. The strain tensor, $\epsilon$ is given by:

$$
\epsilon_{i j}=\frac{1}{2}\left(u_{i, j}+u_{j, i}\right)
$$

A compact representation of the stress tensor may be given by $\sigma_{i j}=\mathbf{C}^{i j k l} u_{i, j}$, where $\mathbf{C}^{i j k l}$ is the stiffness tensor. We note that the acceleration term in (1) is only used when solving the equations of linear elasticity as a structural model; this term is omitted when using the linear elasticity approach for computing mesh deformations. When applying the linear elasticity model for mesh deformation the parameters $E$ and $\nu$ may be varied spatially to improve mesh quality. A common choice, also employed here, is to fix $\nu$ and vary $E$ on each element proportionally with the inverse of the Jacobian of mapping from reference to physical space. ${ }^{11}$ Alternatively, $E$ is given as a prescribed function of the physical coordinates of the original mesh. More sophisticated approaches to define the stiffness matrix are possible, ${ }^{11,16}$ though we have yet to apply these techniques.

We define the discretization of the space-time domain as follows. The spatial domain, $\Omega$, is partitioned into non-overlapping elements, $\kappa$, while the time is partitioned into time intervals (time-slabs), $I^{n}=\left[t^{n}, t^{n+1}\right]$. Define $\mathcal{X}=\left\{\mathcal{X} \in \mathcal{H}_{1}(\Omega \times I),\left.\mathcal{X}\right|_{\kappa} \in[\mathcal{P}(\bar{\kappa} \times I)]^{4}\right\}$, the 4-dimensional coordinates the space-time finite-element mesh, consisting of piece-wise polynomial in both space and time on each element. For each time slab, we fix the temporal coordinate and solve for the 3 -spatial coordinates of the displacement of the space-time mesh from a reference initial spatial mesh.

We apply a continuous finite-element discretization of (1) over the initial mesh. Define $\left.\mathcal{V}=\left\{\boldsymbol{w} \in \mathcal{H}_{1}(\Omega) \times \mathcal{L}_{2}(I)\right),\left.\boldsymbol{w}\right|_{\kappa} \in[\mathcal{P}(\kappa \times I)]^{3}\right\}$, the space-time finite-element space consisting of $\mathcal{C}^{0}$ continuous piece-wise polynomial functions on each element.

We seek solutions for the mesh velocity, $\mathbf{v} \in \mathcal{V}_{E}$ satisfying

$$
\sum_{\kappa}\left\{-\int_{I} \int_{\kappa^{0}} \boldsymbol{w}_{i, j} \mathbf{C}^{i j k l} \boldsymbol{u}_{k, l}+\int_{I} \int_{\partial \kappa^{0} \cap \partial \Omega} \boldsymbol{w}_{i} \widehat{\mathbf{C}^{i j k l} \boldsymbol{u}_{k, l}} \boldsymbol{n}_{j}\right\}=0 .
$$


where the displacement is computed as $\boldsymbol{u}(t)=\boldsymbol{u}(0)+\int_{0}^{T} \mathbf{v} d t$. We note that the integration is performed over the initial spatial mesh (i.e elements $\kappa^{0}$ ) of the domain extruded in time, as opposed to the deformed mesh. An alternative approach is to integrate over the initial spatial mesh for each time-slab, which may potentially allow for larger mesh deformations. However, this later approach results in a scheme where the mesh deformation is a function of not only the given boundary displacement but the entire displacement history. We can strongly enforce the specified displacements on the boundaries of the fluid mesh by prescribing the desired values of the boundary degrees of freedom and removing the associated degrees of freedom from $\mathcal{V}_{E}$.

Using a particular choice of basis to discretize the space $\mathcal{V}$, equation (4) leads to a system of linear equations which must be solved for the mesh displacement. Since our goal is to use the mesh deformation strategy as part of an unsteady fluid-structure interaction simulation, efficient solution of the resulting system of equations is required. In this work we use the modified tensor-product formulation of Karniadakis and Sherwin. ${ }^{23}$ The tensor-product formulation allows for evaluation of the residual terms which scale as $N^{d+1}$ where $N$ is the polynomial order, while $d$ is the space-time dimension (i.e. 4 for 3 -D + time). We take advantage of the efficient tensor-product evaluation for the solution of the linear system by using a Jacobian-free Krylov scheme, which does not explicitly require forming the linear system; only the repeated evaluation of the residual. With increasing polynomial-order the linear system corresponding to equation (4) becomes increasingly stiff, requiring a large number of Krylov vectors to solve the corresponding linear system. For the initial implementation discussed here, we have not employed any preconditioning strategy. We recognize that for more difficult problems preconditioning is key to maintain efficiency of the scheme and we have begun working on a preconditioner based on an approximate local element-wise solve using the fast-diagonalization method (FDM $)^{24}$ and a coarse global solve corresponding to either a 2nd- or 3rd- order discretization of the linear elastic problem. Details of the preconditioning strategy and an assessment of its effectiveness will be presented in a future paper.

\section{Mesh Deformation Results}

We present some initial numerical results using our linear-elastic mesh deformation technique. We first consider several steady-state deformation problems, where we solve for the displacement field. Then we demonstrate the space-time elasticity solve the velocity field in order to generate higher-order space-time meshes.

\section{A. Boundary Roughness on channel}

In the first test case we consider using the linear-elasticity approach to generate meshes with higher-order curved boundaries given an initial linear mesh. In particular we use the elasticity approach to deform a linear mesh near the boundary of a channel in order to simulate surface roughness. Sinusoidal displacement of the channel surfaces is applied with varying depth and frequency of surface roughness. We solve for an 8th-order displacement to generate the deformed mesh. In Figure 1 we present two meshes (split left/right) corresponding to displacements with different frequency of surface oscillations. Due to the high frequency of the oscillations (1/4-1/3 times the Nyquist frequency for this mesh) the mesh quality "looks" poor when plotting only the nodal values. Thus, we also over-sample the 8th-order geometry representation from the mesh, from which it is evident that we recover a smooth representation of the boundary displacements.

In Figure 1 we also present a quantitative assessment of mesh quality by plotting the scaled Jacobian of the deformed mesh. The elasticity based deformation results in a high quality mesh with a scaled Jacobian greater than 0.75 everywhere in the domain domain. Figure 2 shows similar results using two different depths of displacements at a fixed frequency. It is evident from the mismatch between the meshes for the shallow (left) and deep (right) displacements that the perturbations extend throughout the domain quite far from the boundary. Again, high-quality elements are generated by the linear-elasticity approach.

\section{B. Low Pressure Turbine Cascade}

The second test case we consider is using the elasticity approach to generate deformed surface meshes for a low pressure turbine (LPT) blade in order to simulate surface roughness. Starting with an initial 8th order 


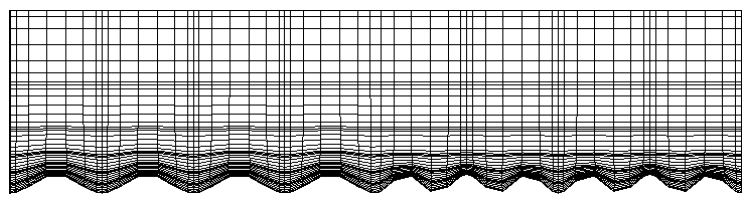

(a) Mesh nodes

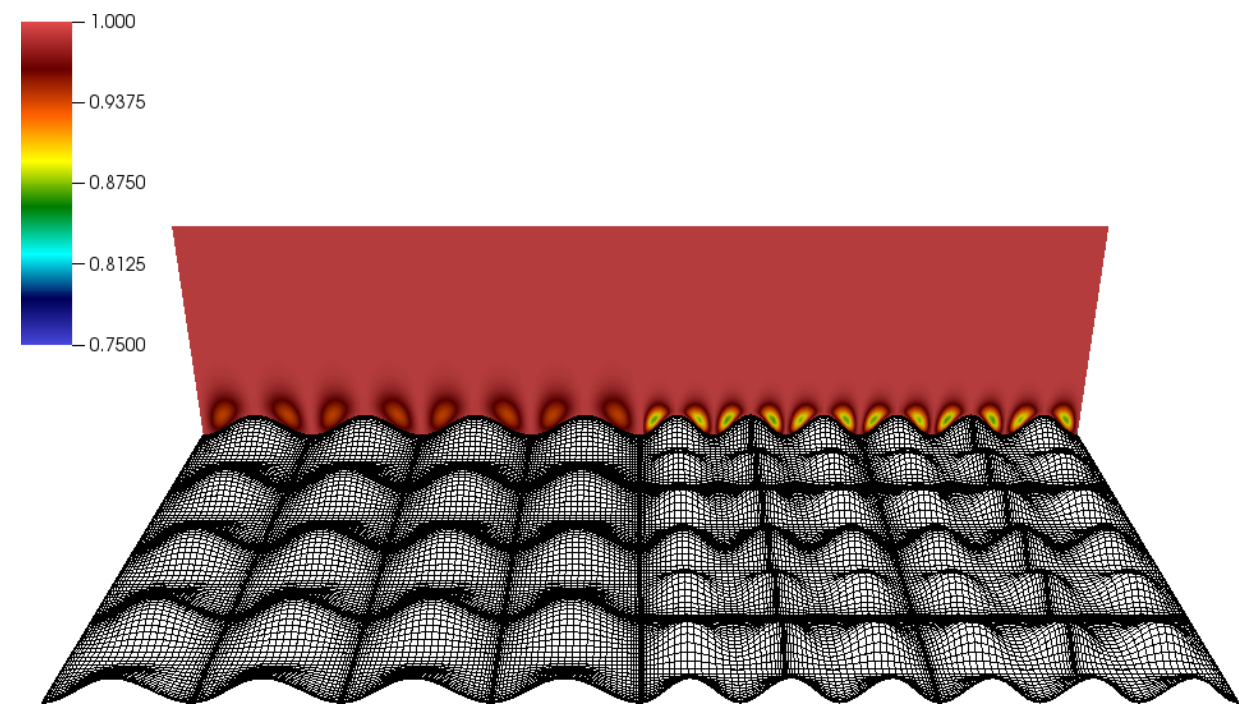

(b) Oversampled mesh and scaled Jacobian

Figure 1. Displaced channel mesh for different frequency perturbations.

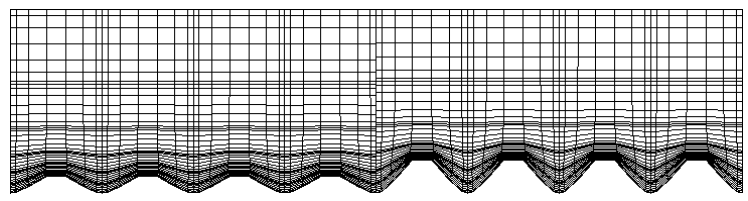

(a) Mesh nodes

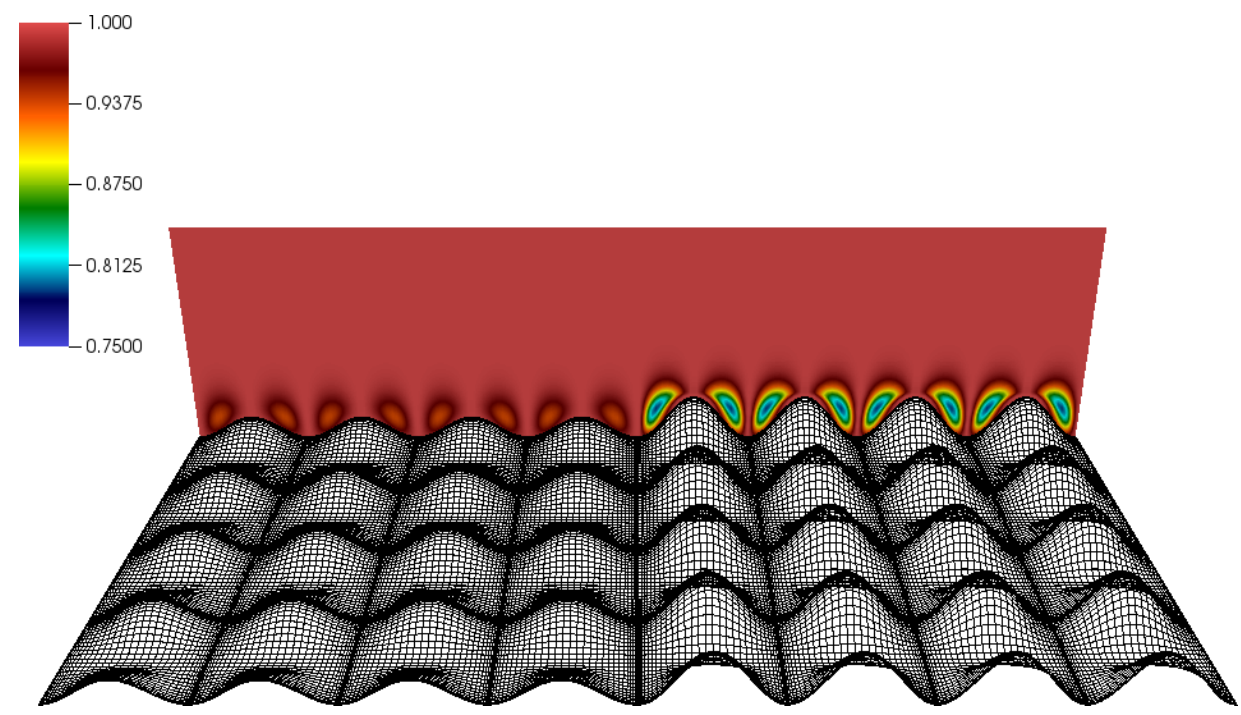

(b) Oversampled mesh and scaled Jacobian

Figure 2. Displaced channel mesh for different depth perturbations. 
curvilinear mesh surface normal purtubations are applied and we use the elasticity approach to propogate the surface deformations into the domain. Once again we use the elasticity approach to solve for a temporally fixed mesh displacement. Figure 3 shows the deformation applied at the surface of the turbine blade, as well as the deformed mesh near the leading edge of the blade.

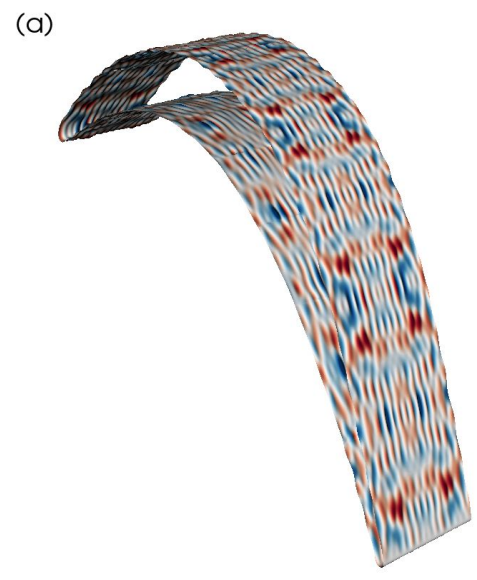

(a) Surface Deformation

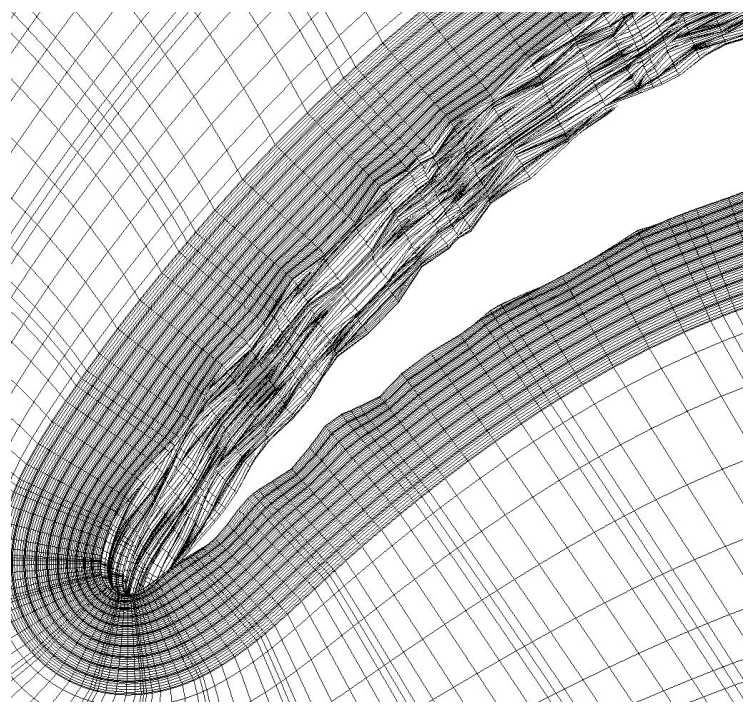

(b) Deformed Mesh

Figure 3. Surface deformation and deformed mesh for LPT blade

The perturbed mesh is used to discretize the compressible Navier-Stokes equations (details of the equations solved are given in Section IV). Figure 4 shows snapshots of the corresponding Navier-Stokes simulations using the unperturbed and perturbed geometries.

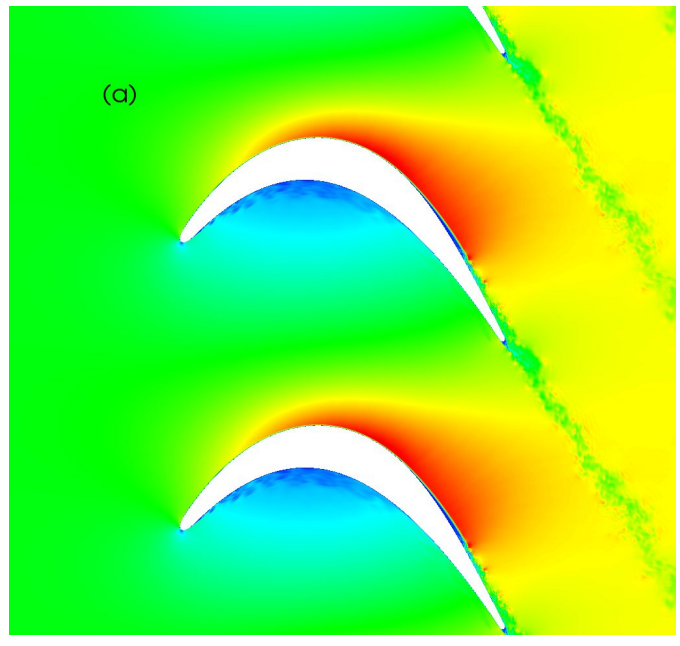

(a) Smooth blade

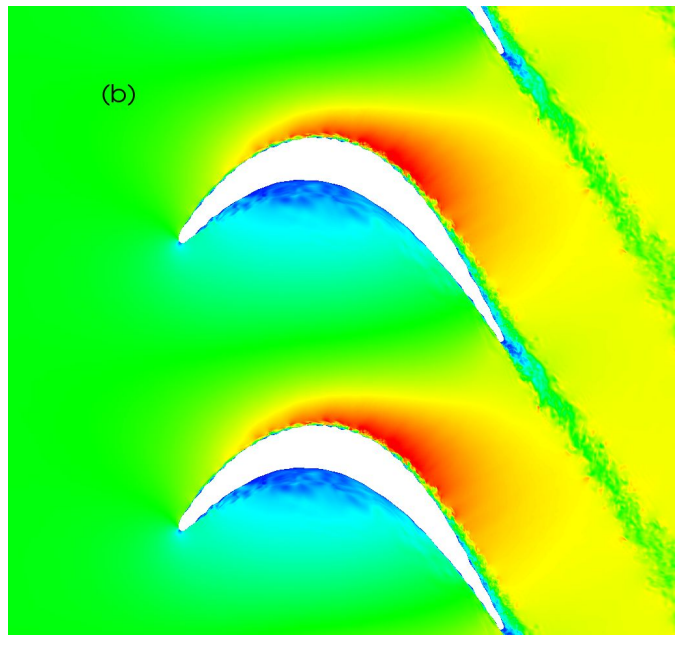

(b) Blade w/ Surface Roughness

Figure 4. Snapshot of Mach number from Navier-Stokes simulations of LPT blade with and without surface roughness

In the baseline smooth configuration a small separation bubble is evident on the suction side of the blade. With simulated surface roughness, the flow remains attached over the entire blade matching the experimentally observed behaviour. Complete details of this test case and the effects of surface roughness are presented in an upcoming article. ${ }^{25}$ 


\section{Rotating Box}

The third test case we consider is a small box rotated within a larger box. This test case was previously used by Froehle and Persson ${ }^{22}$ to assess a nonlinear elasticity approach for mesh deformation. This test case is intended to stress the elasticity approach to determine how large displacements may be applied while still generating a valid mesh. The test case which involves embedding a box of size $(0.2 \times 0.2)$ within a unit box $(1 \times 1)$ and rotating the interior box until the resulting deformed mesh has an invalid element. We consider displacing 2nd, 4th and 8th order meshes with a fixed number of degrees of freedom. First we consider the linear-elastic approach using a spatially constant Young's modulus. Figure 5 shows initial 2nd, 4th and 8th order meshes and the corresponding deformed meshes with rotation of $15^{\circ}$ and $30^{\circ}$. We colour the deformed meshes using the scaled determinant of the deformed mesh. Using a spatially constant Young's modulus, there is significant skewing of the elements near the corners of the inner box already at $15^{\circ}$. At rotation angles greater than $20^{\circ}$, invalid 8th order meshes are generated with inverted elements at the corners of the interior box. At lower orders, somewhat larger rotation angles may be applied while maintaining valid elements. However, even at 2nd order, valid meshes cannot be generated with rotation angles greater than $35^{\circ}$.

Next we consider the spatially varying Young's modulus used by Froehle and Persson. ${ }^{22}$ The Young's modulus is given by:

$$
E(x)=1+\frac{100}{1+\left(d(x) / d_{0}\right)^{2}}
$$

where $d_{0}=0.05$ and $d(x)=\max \left\{0.0, \min \left(\operatorname{dist}\left(x, \Gamma_{\text {in }}\right)-d_{0}, \operatorname{dist}\left(x, \Gamma_{\text {out }}\right)+2 d_{0}\right)\right\}$. Here $\Gamma_{\text {in }}$ and $\Gamma_{\text {out }}$ are respectively the inner and outer boundaries of the domain, while $\operatorname{dist}\left(x, \Gamma_{i}\right)$ denotes the distance to the boundary. With this spatially varying Young's modulus the linear-elastic approach allows for much larger mesh deformations than a constant Young's modulus. Figure 6 shows the deformed mesh and corresponding determinant of the scaled Jacobian at rotations of $15^{\circ}, 30^{\circ}$ and $45^{\circ}$. By increasing the stiffness near the boundaries, essentially rigid body deformation occurs near the boundaries while larger deformations occur on the interior of the domain. With the variable coefficient, the elasticity approach is able to generate valid 2nd and 4th order meshes with a rotation of $45^{\circ}$, while at 8 th order a valid mesh is generated for rotations of up to $40^{\circ}$. We note that using a nonlinear elasticity approach, even larger displacements may be possible, ${ }^{22}$ however the impact of the Young's modulus is much more significant than switching to a nonlinear approach. In particular, we found that using the nonlinear approach with a constant Young's modulus allowed for significantly less rotation than simply changing the Young's modulus. Additionally switching to a nonlinear approach make the resulting system extremely stiff and difficult to solve. ${ }^{19}$

\section{Heaving/Pitching Airfoil}

The fourth test case we consider is generating a higher-order space-time mesh for a moving-body simulation. In particular, we consider one of the test cases from the Higher-Order Workshop. ${ }^{18}$ The test case involves flow over the NACA0012 airfoil at $M=0.2, R e=5000$ initially at zero angle of attack with a prescribed heaving/pitching motion. In this case we solve for the space-time mesh velocity and recover the displacement by integrating the mesh velocity. Figure 7 depicts the initial 4 th order mesh, along with the space-time mesh deformed using elasticity at several temporal locations throughout the heaving/pitching motion. Displacing the interior nodes of the mesh the linear elastic model, with Poisson ratio of $\nu=0.4$ and Young's modulus scaled with the inverse of the Jacobian determinant, ensures valid elements everywhere in the domain despite the large deformations due to the prescribed motion. We quantify the quality of the mesh by plotting the determinant of the deformation gradient tensor near the surface of the airfoil. As can be seen, the linearelastic model maintains high quality elements throughout the domain with the deformation tensor near 1 everywhere in the domain except for some significant deformation near the trailing edge. We note as in the previous test case, that using a constant Young's modulus throughout the domain results in invalid elements. Similarly, using a Young's modulus scaled inversely with the Jacobian determinant ensures near rigid body motion near the airfoil surface as we generally have small elements here, while larger deformations are allowed further from the airfoil surface where there are larger elements. 


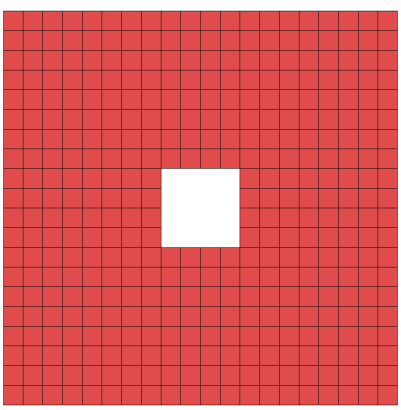

(a) $N=2, \theta=0^{\circ}$

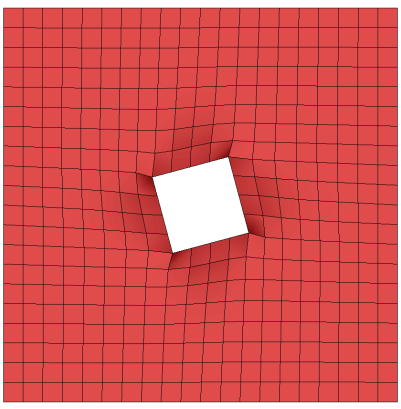

(d) $N=2, \theta=15^{\circ}$

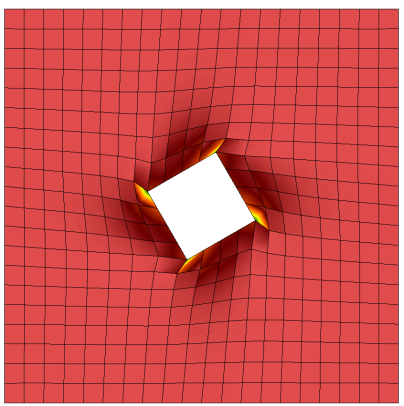

(g) $N=2, \theta=30^{\circ}$

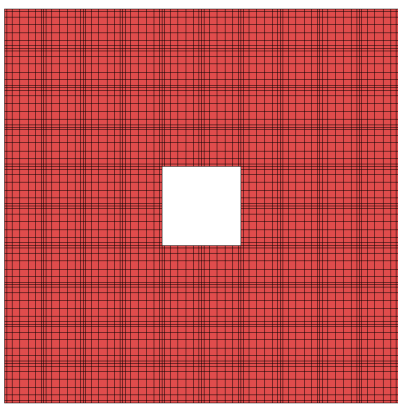

(b) $N=4, \theta=0^{\circ}$

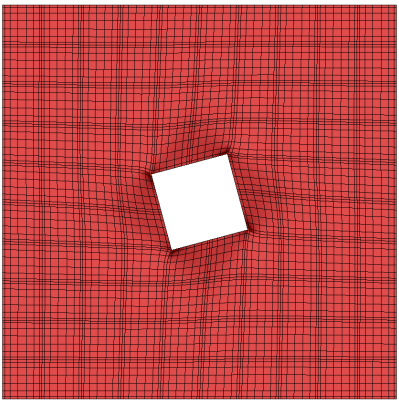

(e) $N=4, \theta=15^{\circ}$

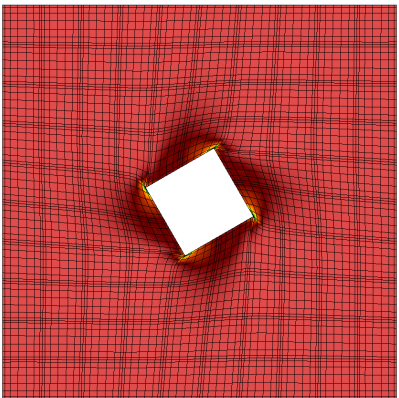

(h) $N=4, \theta=30^{\circ}$

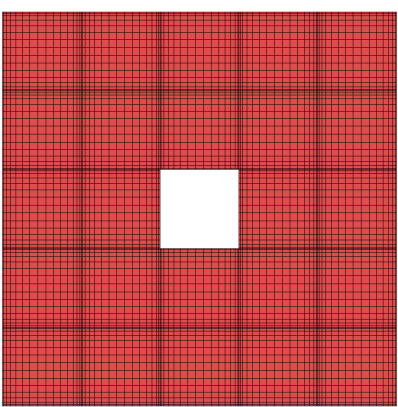

(c) $N=8, \theta=0^{\circ}$

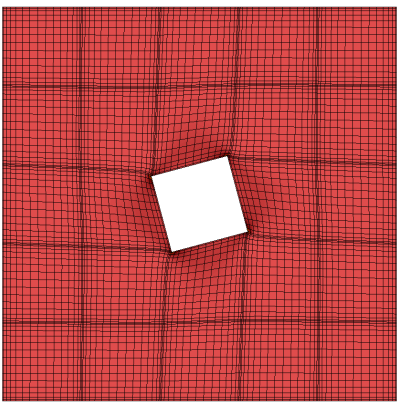

(f) $N=8, \theta=15^{\circ}$

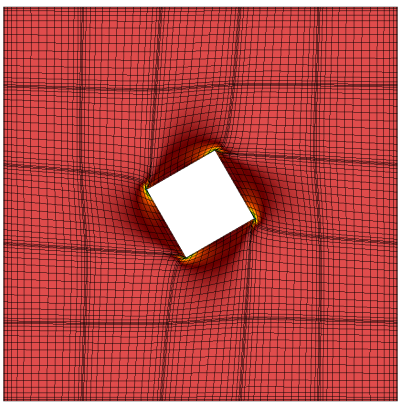

(i) $N=8, \theta=30^{\circ}$

Figure 5. Rotating box test case using constant Young's modulus for different orders and angles of rotation. Meshes coloured by determinant of scaled Jacobian. (Note: for $N=4$ and $N=8$, grid depicts 2N quadrature points on each element) 


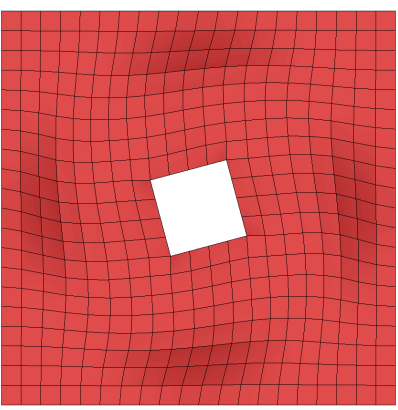

(a) $N=2, \theta=15^{\circ}$

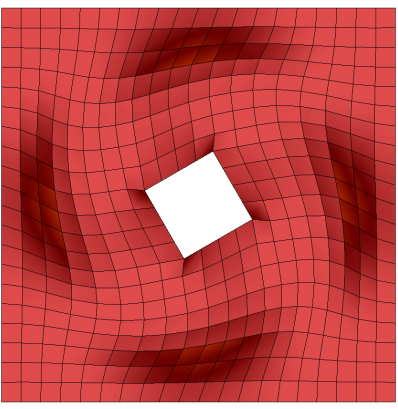

(d) $N=2, \theta=30^{\circ}$

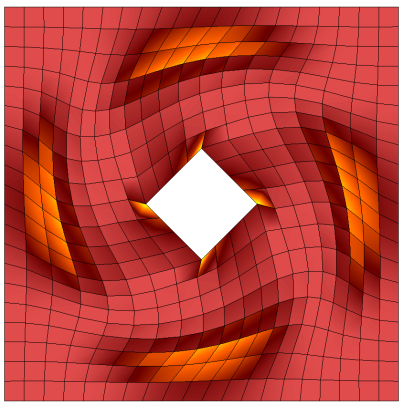

(g) $N=2, \theta=45^{\circ}$

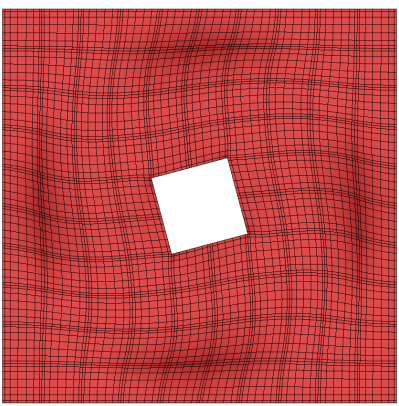

(b) $N=4, \theta=15^{\circ}$

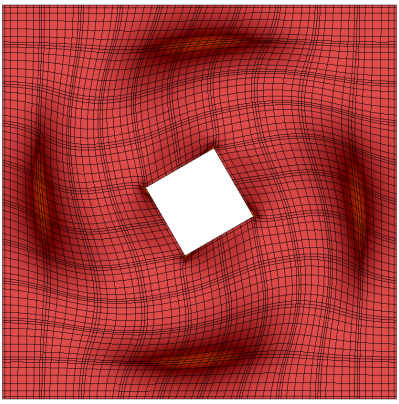

(e) $N=4, \theta=30^{\circ}$

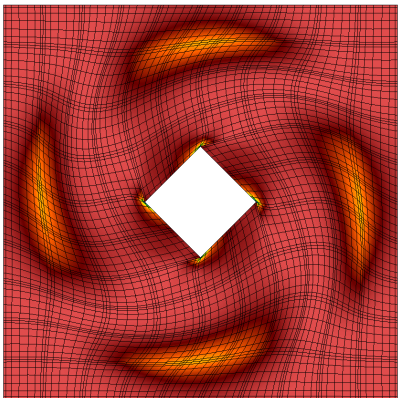

(h) $N=4, \theta=45^{\circ}$

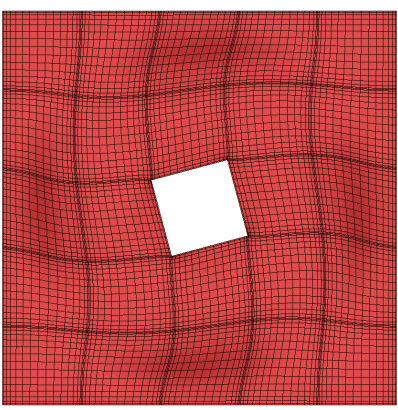

(c) $N=8, \theta=15^{\circ}$

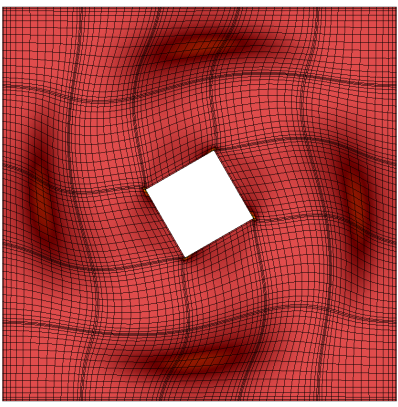

(f) $N=8, \theta=30^{\circ}$

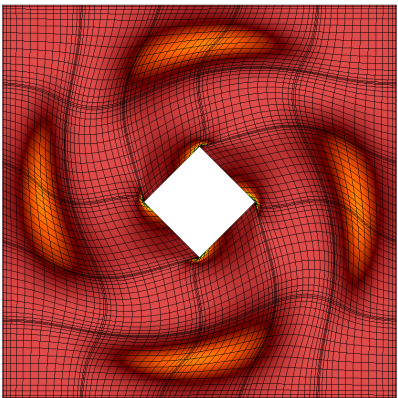

(i) $N=8, \theta=45^{\circ}$

Figure 6. Rotating box test case using spatially varying Young's modulus for different orders and angles of rotation. Meshes coloured by determinant of scaled Jacobian. (Note: for $N=4$ and $N=8$, grid depicts 2N quadrature points on each element) 


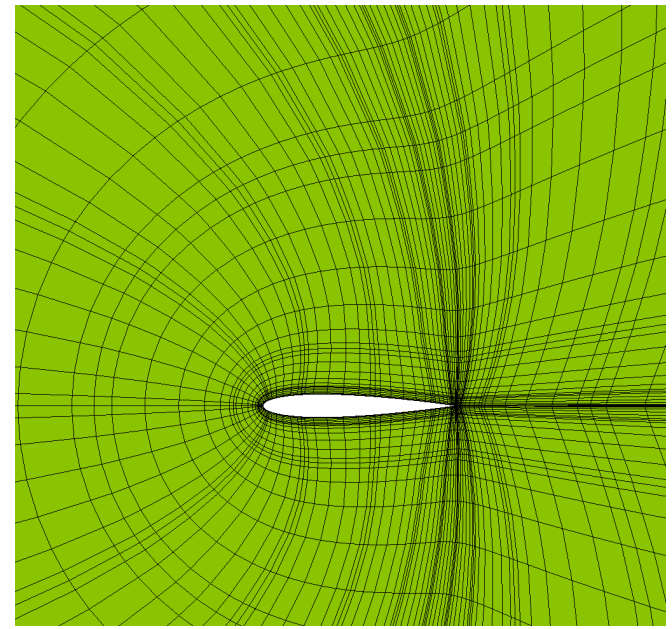

(a) $t=0.0$

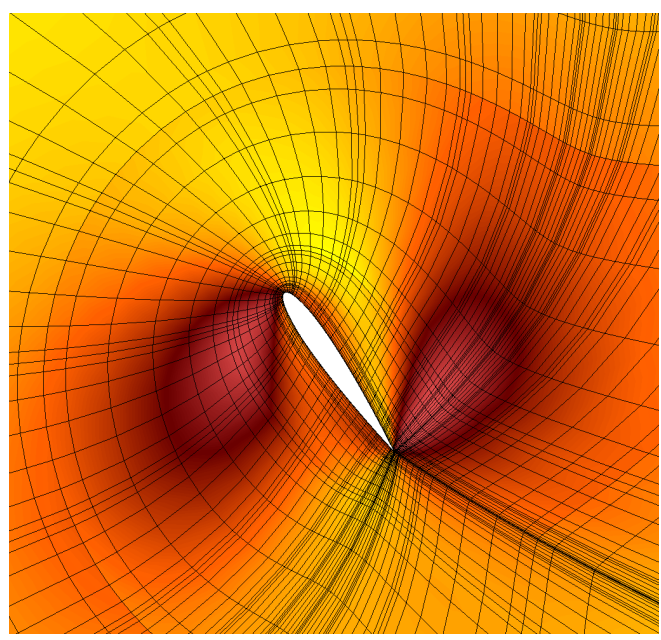

(c) $t=0.8$

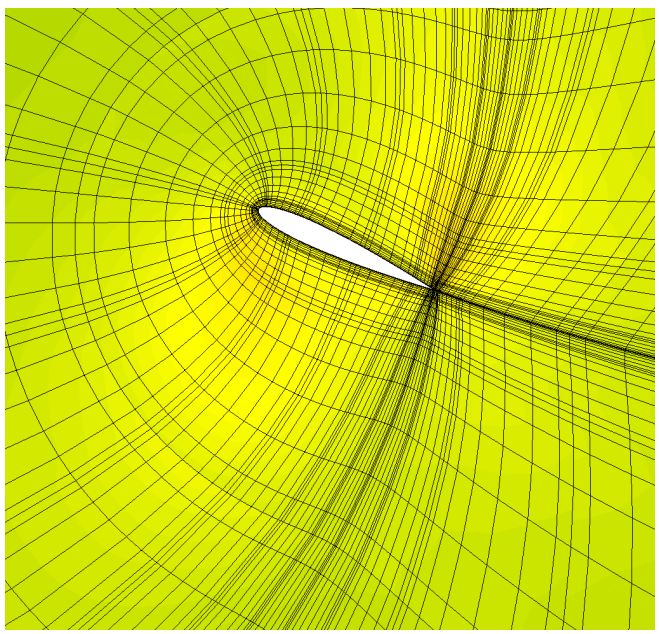

(e) $t=1.6$

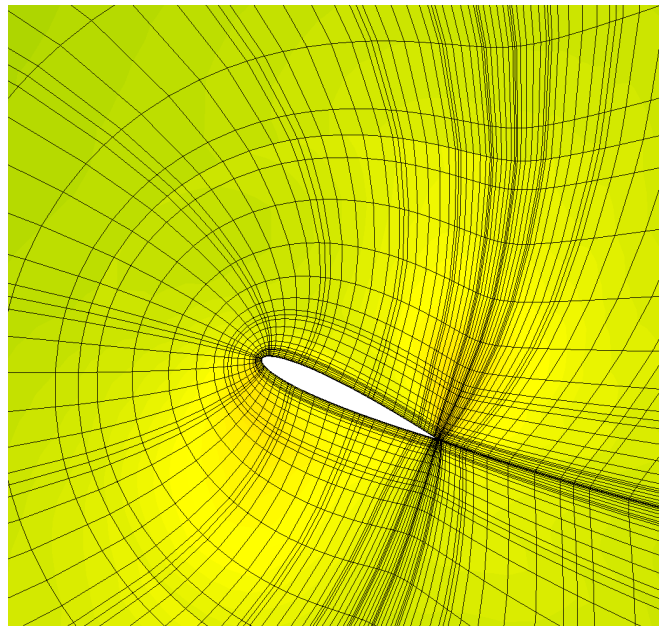

(b) $t=0.4$

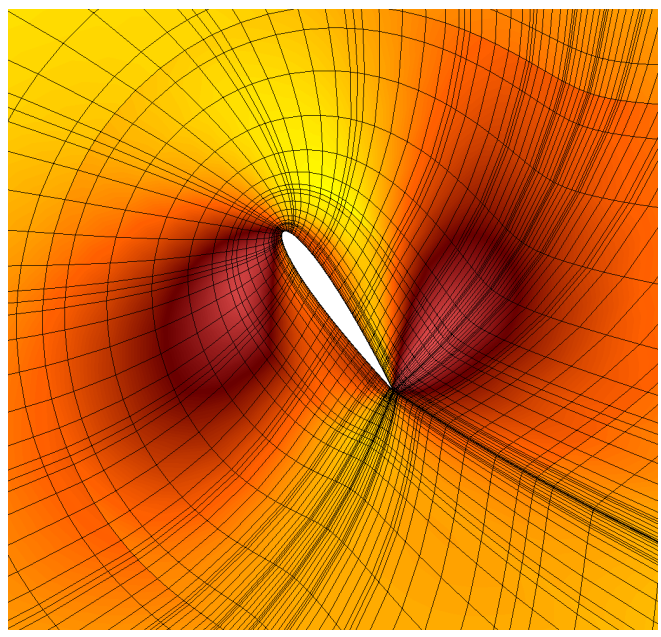

(d) $t=1.2$

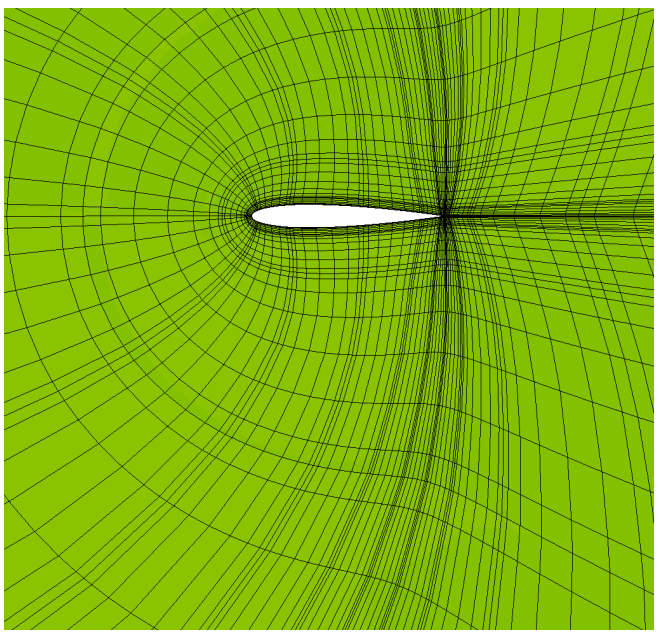

(f) $t=2.0$

Figure 7. Heaving/Pitching Airfoil test case: Initial and displaced mesh at various temporal locations coloured with determinant of the deformation gradient tensor 


\section{Coupled Results}

In this section we couple the linear-elasticity approach with a compressible Navier-Stokes solver for fluid dynamics simulations. ${ }^{26}$ For the fluid domain we solve the compressible Navier-Stokes equations written in conservative form as

$$
\boldsymbol{u}_{, t}+\left(\boldsymbol{F}^{I n v}-\boldsymbol{F}^{V i s c}\right)_{, i}=0,
$$

where $\boldsymbol{u}=\left[\rho, \rho u_{j}, \rho E\right]$ is the conservative state vector, with $\rho$ the density of the fluid, $u_{j}$ the velocity components and $E$ the total energy. The inviscid and viscous fluxes are given respectively by

$$
\boldsymbol{F}^{I n v}=\left[\begin{array}{c}
\rho u_{i} \\
\rho u_{j} u_{i}+p \delta_{i j} \\
\rho H u_{i}
\end{array}\right] \quad \boldsymbol{F}^{V i s c}=\left[\begin{array}{c}
0 \\
\tau_{i j} \\
\tau_{i j} u_{j}+\kappa_{T} T_{, j}
\end{array}\right]
$$

where $p$ is the static pressure, $\delta_{i j}$ the Kronecker delta, $H$ the total enthalpy, $\tau_{i j}$ the viscous stress tensor, $T$ the temperature and $\kappa_{T}$ the thermal conductivity. The system is closed using the following relationships

$$
T=\frac{p}{\rho R}, \quad p=(\gamma-1)\left(\rho E-\frac{1}{2} \rho u_{k} u_{k}\right), \quad \tau_{i j}=\mu\left(u_{i, j}+u_{j, i}\right)-\lambda u_{k, x_{k}} \delta_{i j},
$$

where $R$ is the gas constant, $\gamma$ the specific heat ratio, $\mu$ the dynamic viscosity and $\lambda=\frac{2}{3} \mu$ the bulk viscosity.

We use a space-time discontinuous-Galerkin discretization of (6). We seek a solution $\mathbf{u}$ which satisfies the weak form

$\sum_{\kappa}\left\{\int_{I} \int_{\kappa}-\left(\mathbf{w}_{, t} \mathbf{u}+\mathbf{w}_{, i}\left(\boldsymbol{f}_{i}^{I}-\boldsymbol{f}_{i}^{V}\right)\right)+\int_{I} \int_{\partial \kappa} \mathbf{w}\left(\widehat{\boldsymbol{f}_{i}^{I} \boldsymbol{n}_{i}}-\widehat{\boldsymbol{f}_{i}^{V} \boldsymbol{n}_{i}}\right)+\int_{\kappa} \mathbf{w}\left(t_{-}^{n+1}\right) \mathbf{u}\left(t_{-}^{n+1}\right)-\mathbf{w}\left(t_{+}^{n}\right) \mathbf{u}\left(t_{-}^{n}\right)\right\}=0$

where the second and third integrals arise due to the spatial and temporal discontinuity, respectively, of the basis functions. $\widehat{\boldsymbol{f}_{i}^{I} \boldsymbol{n}_{i}}$ and $\widehat{\boldsymbol{f}_{i}^{V} \boldsymbol{n}_{i}}$ denote single valued numerical flux functions approximating, respectively, the inviscid and viscous fluxes at the spatial boundaries of the elements.

In a moving-body simulations there is only one-way coupling between the elasticity domain and the fluid domain. We prescribe the motion, thus the elasticity solve is independent of the fluid domain. However, the fluid domain depends implicitly on the mesh deformation, as the integrals in (9) are over the deformed mesh. In the future we anticipate using this approach for performing fluid-structure interation, where the boundary deformation will be prescribed by a structural model, which in turn will depend on the forces exerted by the fluid domain. Thus for this test case we solve the coupled elasticity/fluid problem simultaneously.

\section{A. Isentropic Vortex Convection}

In this test case we consider the isentropic convection of a vortex, while deforming the mesh with a prescribed motion. The isentropic vortex convection problem is initialized with a perturbation about a uniform flow given by:

$$
\begin{aligned}
\delta u & =-U_{\infty} \beta \frac{y-y_{c}}{R} e^{-\frac{r^{2}}{2}} \\
\delta v & =U_{\infty} \beta \frac{x-x_{c}}{R} e^{-\frac{r^{2}}{2}} \\
\delta\left(\frac{P}{\rho}\right) & =\frac{1}{2} \frac{\gamma}{\gamma-1} U_{\infty}^{2} \beta^{2} e^{-r^{2}}
\end{aligned}
$$

where $U_{\infty}$ is the convecting speed of the vortex, $\beta$ is the vortex strength, $R$ the characteristic radius, and $\left(x_{c}, y_{c}\right)$ the vortex center. The exact solution to this flow is a pure convection of the vortex at speed $U_{\infty}$.

We consider a moving-body simulation with a mesh deformed to track the convection of the vortex and deformed sinusoidally with boundary displacement given by:

$$
\begin{aligned}
& x^{\prime}=x+0.1\left(x-x_{c}\right) \sin \left(\frac{\pi}{4} t\right)+U_{\infty} t \\
& y^{\prime}=y-0.1\left(y-y_{c}\right) \sin \left(\frac{\pi}{4} t\right) \nu(1-\nu)
\end{aligned}
$$



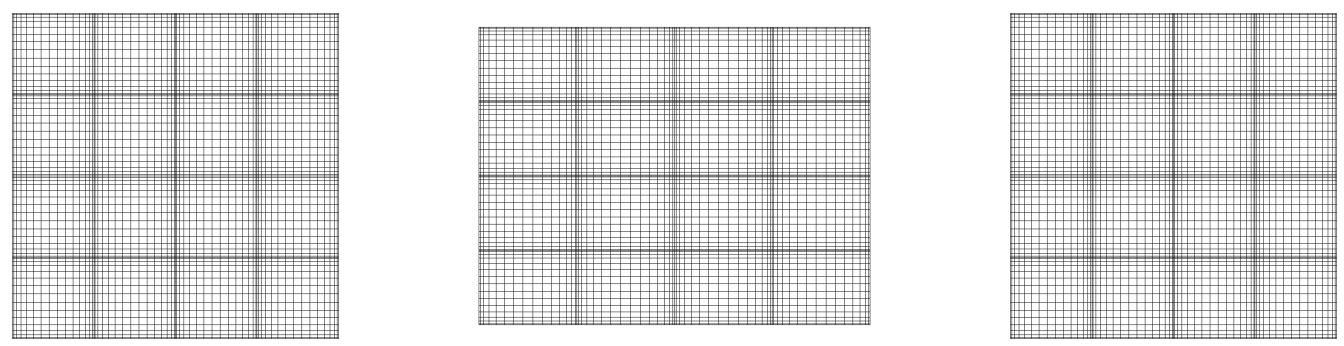

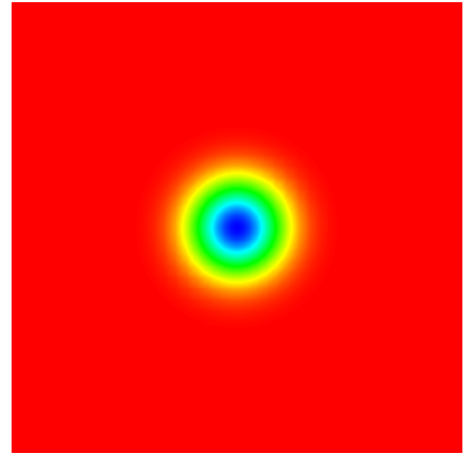

(a) $t=0.0$

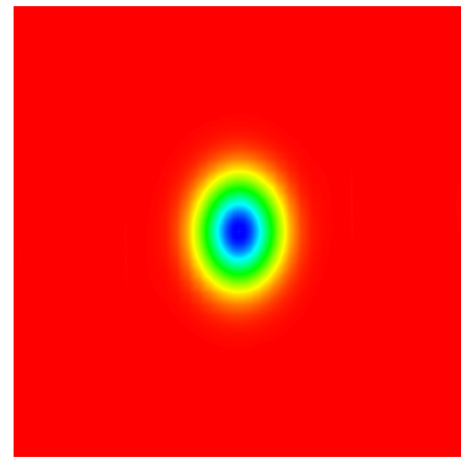

(b) $t=2.5$

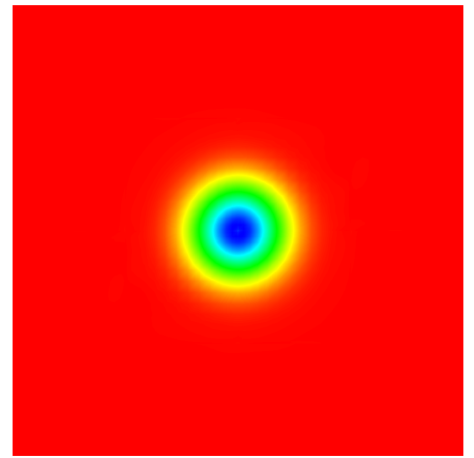

(c) $t=5.0$
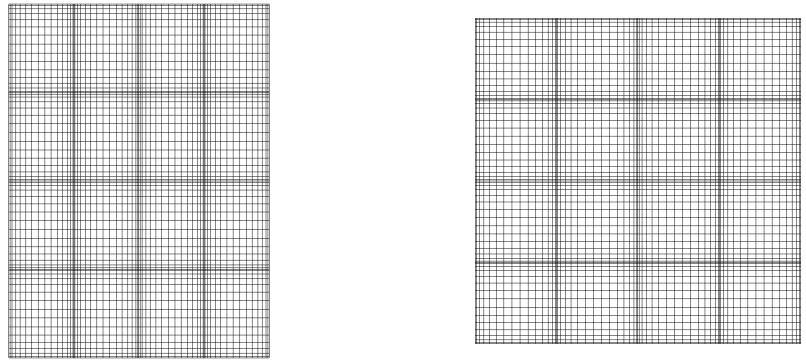

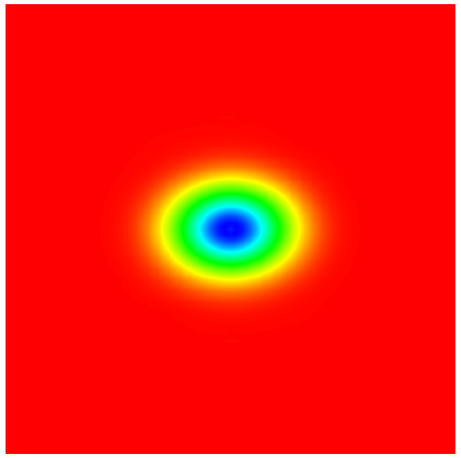

(d) $t=7.5$

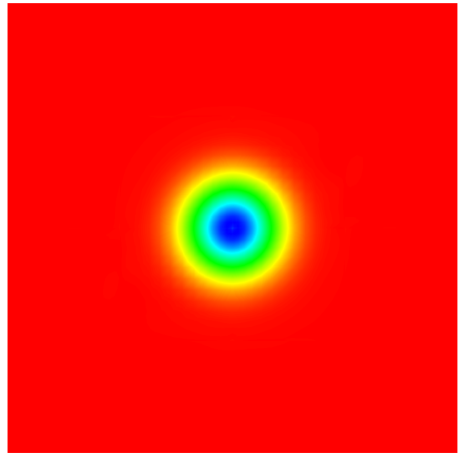

(e) $t=10.0$

Figure 8. Deformed mesh and fluid solution on original mesh for isentropic vortex convection problem 
Figure 8 depicts the displaced mesh through one cycle of deformation and the corresponding vortex plotted in the original (as opposed to the displaced) coordinate system. As can be seen, the coupled spacetime finite-element method maintains a high-quality solution through one cycle of mesh motion.

We note that solving the coupled elasticity-fluid problem is significantly harder than solving the elasticity or the fluid independently. There are several challenges in solving the coupled problem that need to be addressed before this methodology can be applied to more complex problems. In particular, we note that the stiffness of the space-time elasticity problem is independent of the time-step (as we drop all temporal terms this is essentially a "steady" solve), while the stiffness of the fluid solve decreases with time step. Thus even for very small time-steps the elasticity solve requires efficient preconditioning to be practical. Furthurmore, partially converged elasticity solutions may result in mesh deformations with invalid elements (even if the converged solution is valid), resulting in non-physical residual evaluations on the fluid domain. Thus a sophisticated globalization strategy is required. Currently, a pseudo-transient type globalization strategy is employed for the fluid domain, while no globalization strategy is used for the elasticity domain. The combination of the different preconditioning and globalization strategies on the two domains results in some non-intuitive convergence behaviour such as poorer convergence when decreasing the time step. It is anticipated that improving the preconditioning and extending the globalization strategy for the elasticity domain will significantly improve the solver performance.

\section{Outlook/Future Work}

In this paper we have presented a linear elastic solution strategy for higher-order space-time mesh deformation, and demonstrated the application of this strategy for some simple problems. The elasticity approach was shown to be appropriate for generating higher-order meshes for fixed-grid simulations and higher-order space-time meshes for use in moving-body fluid dynamics simulations. In our initial approach we have applied a very simple solution strategy which involved no preconditioning or globalization. Application of this approach for coupled fluid-structure applications will require addressing these limitations. Future applications will focus on coupling the fluid solve and mesh deformation to a structural model.

\section{References}

${ }^{1}$ Diosady, L. T. and Murman, S. M., "Design of a variational multiscale method for turbulent compressible flows," AIAA Paper 2013-2870, 2013.

${ }^{2}$ Diosady, L. T. and Murman, S. M., "DNS of flows over periodic hills using a discontinuous Galerkin spectral element method," AIAA Paper 2014-2784, 2014.

${ }^{3}$ Diosady, L. T. and Murman, S. M., "Tensor-Product Preconditioners for Higher-order Space-Time Discontinuous Galerkin Methods," 2014, under review.

${ }^{4}$ Diosady, L. T. and Murman, S. M., "Higher-Order Methods for Compressible Turbulent Flows Using Entropy Variables," AIAA Paper 2015-0294, 2015.

${ }^{5}$ Ceze, M., Diosady, L., and Murman, S., "Development of a High-Order Space-Time Matrix-Free Adjoint Solver," AIAA 2016-0833, 2016.

${ }^{6}$ Burgess, N. K., Diosady, L. T., and Murman, S. M., "A C1-discontinuous-Galerkin Spectral-element Shell Structural Solver," AIAA Paper 2017-3727, 2017.

${ }^{7}$ Hubner, B., Walhorn, E., and Dinkler, D., "A monolithic approach to fluid-structure interaction using space-time finite elements," Comput. Methods Appl. Mech. Engrg., Vol. 2004, 2004, pp. 2087-2104.

${ }^{8}$ Klaij, C., van der Vegt, J., and van der Ven, H., "Spacetime discontinuous Galerkin method for the compressible NavierStokes equations," Journal of Computational Physics, Vol. 217, 2006, pp. 589611.

${ }^{9}$ Heil, M., Hazel, A. L., and Boyle, J., "Solvers for large-displacement fluidstructure interaction problems: segregated versus monolithic approaches," Computational Mechanics, Vol. 43, 2008, pp. 91-101.

${ }^{10}$ van Brummelen, E., Hulshoff, S., and de Borst, R., "A monolithic approach to fluid-structure interaction using space-time finite elements," Comput. Methods Appl. Mech. Engrg., Vol. 2003, 2003, pp. 2727-2748.

${ }^{11}$ K. Stein, T. T. and Benney, R., "Mesh Moving Techniques for Fluid-Structure Interactions With Large Displacements," J. Appl. Mech, Vol. 70, No. 1, 2003, pp. 58-63.

${ }^{12}$ Tezduyar, T. E. and Sathe, S., "Modelling of fluid-structure interactions with the space-time finite elements: Solution techniques," Int. J. Numer. Meth. Fluids, Vol. 54, 2007, pp. 855-900.

${ }^{13}$ Wang, L. and Persson, P.-O., "A high-order discontinuous Galerkin method with unstructured space-time meshes for two-dimensional compressible flows on domains with large deformations," Computers and Fluids, Vol. 118, 2015, pp. 53-68.

${ }^{14}$ Lesoinne, M. and Farhat, C., "Geometric conservation laws for flow problems with moving boundaries and deformable meshes, and their impact on aeroelastic computations," Comput. Methods Appl. Mech. Engrg., Vol. 134, 1996, pp. 71-90.

${ }^{15}$ Yano, M., An Optimization Framework for Adaptive Higher-Order Discretizations of Partial Differential Equations on Anisotropic Simplex Meshes, Ph.D. thesis, MIT, 2009. 
${ }^{16}$ Yang, Z. and Mavriplis, D. J., "A Mesh Deformation Strategy Optimized by the Adjoint Method on Unstructured meshes," AIAA Paper 2007-0557, 2007.

${ }^{17}$ Barth, T. J., "Numerical Methods for Gasdynamic Systems on Unstructured Meshes," An Introduction to Recent Developments in Theory and Numerics for Conservation Laws, edited by D. Kroner, M. Olhberger, and C. Rohde, Springer-Verlag, 1999, pp. $195-282$.

${ }^{18}$ Wang, Z., Fidkowski, K., Abgrall, R., Bassi, F., Caraeni, D., Cary, A., Deconinck, H., Hartmann, R., Hillewaert, K., Huynh, H., Kroll, N., May, G., Persson, P.-O., van Leer, B., and Visbal, M., "High-Order CFD Methods: Current Status and Perspective," International Journal for Numerical Methods in Fluids, Vol. 72, 2013, pp. 811-845.

${ }^{19}$ Persson, P.-O. and Peraire, J., "Curved Mesh Generation and Mesh Refinement using Lagrangian Solid Mechanics," AIAA Paper AIAA-2009-0949, 2009.

${ }^{20}$ Moxey, D., Ekelschot, D., Keskin, ., Sherwin, S., and Peiro, J., "A Thermo-elastic Analogy for High-order Curvilinear Meshing with Control of Mesh Validity and Quality," Procedia Engineering, Vol. 82, 2014, pp. 127-135.

${ }^{21}$ Moxey, D., Ekelschot, D., Keskin, U., Sherwin, S., and Peiro, J., "High-order curvilinear meshing using a thermo-elastic analogy," Computer-Aided Design, Vol. 72, 2016, pp. 130-139.

${ }^{22}$ Froehle, B. and Persson, P., "Nonlinear Elasticity for Mesh Deformation with High-Order Discontinuous Galerkin Methods for the Navier-Stokes Equations on Deforming Domains," Spectral and High Order Methods for Partial Differential Equations ICOSAHOM 2014, edited by R. Kirby, M. Berzins, and J. Hesthaven, 2015.

${ }^{23}$ Karniadakis, G. and Sherwin, S., Spectral/hp element methods for CFD, Oxford University Press, New York, NY, 1999.

${ }^{24}$ Lynch, R. E., Rice, J. R., and Thomas, D. H., "Direct solution of partial difference equations by tensor product methods," Numerische Mathematik, Vol. 6, No. 1, 1964, pp. 185-199.

${ }^{25}$ Garai, A., Diosady, L., Murman, S., and Madavan, N., "Scale-Resolving Simulations of Low-Pressure Turbine Cascades with Wall Roughness Using a Spectral-Element Method," Proceedings of the ASME Turbo Expo 2018: Turbomachinery Technical Conference and Exposition, No. GT2018-75982, 2018.

${ }^{26}$ Wiart, C. C. D., Diosady, L. T., Garai, A., Burgess, N., Blonigan, P., Ekelschot, D., and Murman, S. M., "Design of a modular monolithic implicit solver for multi-physics applications," AIAA Paper 2018-1400, 2018. 